\title{
SINDROME DE HUNTER: UNA ENFERMEDAD LISOSOMAL POCO FRECUENTE
}

\author{
HUNTER SYNDROME: A RARE LYSOSOMAL DISEASE
}

\author{
Claudia Veralucia Saldaña-Díaz', Fiona Lucía Valencia-Chion', Simón Arturo Venancio-Masgo', \\ Fiorella Lizeth Vilca-Salguero', Miriam Alarcón-Guzmán²,3
}

\begin{abstract}
RESUMEN
Introducción: Las mucopolisacaridosis (MPS) son un grupo de alteraciones metabólicas hereditarias que ocurren por déficit de alguna de las enzimas que degradan los glicosaminoglicanos (GAG). La mucopolisacaridosis tipo II (MPS II), o síndrome de Hunter, es la única MPS con un patrón recesivo ligado al cromosoma X, afectando primariamente a los varones. Se caracteriza por una deficiencia de iduronato-2-sulfatasa (I2S), que contribuye a los signos y síntomas de esta enfermedad, y genera afectación multiorgánica y muerte temprana por acumulación tisular progresiva de los glicosaminoglicanos. Caso Clínico: Paciente varón de 7 años de edad con cuadro de distres respiratorio, que ingresa a Emergencia presentando crisis asmática. Como antecedente tiene diagnóstico hace 6 años de mucopolisacaridosis tipo II. Presenta las siguientes manifestaciones de la enfermedad: dismorfismo facial, piel gruesa, macrocefalia y macroglosia, hernia inguinal y umbilical, hepatoesplenomegalia, retraso mental severo, hipertrofia adenoidea, bronconeumonía y retraso global en el desarrollo psicomotor. Evoluciona favorablemente del episodio asmático, y se le da de alta para control por consultorio. Discusión: El tratamiento del síndrome de Hunter antes era paliativo, actualmente la terapia de reemplazo enzimático abre una nueva posibilidad para tratar a los pacientes con esta condición, mejorando su calidad de vida.
\end{abstract}

Palabras clave: Mucopolisacaridosis II; Iduronato sulfatasa; Terapia de reemplazo enzimático. (fuente: DeCS BIREME)

\begin{abstract}
Introduction: Mucopolysaccharidosis (MPS) are a family of inherited disorders which are caused by a deficiency in the activity of the distinct lysosomal enzymes required for the degradation of the GAGs. Type II mucopolysaccharidosis, also known as Hunter syndrome is the only X-linked MPS, affecting mostly boys. It's caused by a deficiency of the lysosomal enzyme iduronate-2-sulfatase, contributing to the signs and symptoms of the disease, causing a multiorganic disease and early death because of the glycosaminoglycan accumulation. Case Presentation: Seven year old boy with respiratory distress, who comes to the hospital with an asthma crisis. Six years ago, he was diagnosed with Type II Mucopolysaccharidosis. He has the following disease signs: facial dysmorphism, thickened skin, macrocephalus, large tongue, inguinal and umbilical hernia, enlarged liver and spleen, profound mental impairment, hypertrophic adenoids, bronchopneumonia and globally delayed developmental milestones. He was discharged and continued his attention in the hospital. Discussion: The treatment of Hunter Syndrome has been palliative, now Enzyme Replacement Therapy opens new possibilities of treatment, improving the quality of life of the patients.
\end{abstract}

Key words: Mucopolysaccharidosis II; Iduronate sulfatase; Enzyme replacement therapy. (source: MeSH NLM)

\footnotetext{
1 Internado Médico, Hospital Nacional Guillermo Almenara Irigoyen.

${ }^{2}$ Médico asistente del servicio de Neuropediatría del Hospital Nacional Guillermo Almenara Irigoyen.

${ }^{3}$ Facultad de Medicina Humana de la Universidad Ricardo Palma.

Correspondencia: Fiorella Vilca Salguero. Dirección: Jr. Cajamarca 624, Rimac. Celular: 959783448. Correo: fio_lella@hotmail.com
} 


\section{INTRODUCCIÓN}

Las mucopolisacaridosis (MPS) son un grupo de alteraciones metabólicas hereditarias que ocurren por déficit de alguna de las enzimas que degradan estas moléculas en los lisosomas, produciendo el acúmulo de diversos tipos de glicosaminoglicanos (GAG)' , siendo la incidencia global estimada en 1:10000 a 1:25000².

Actualmente la clasificación de la mucopolisacaridosis comprende siete tipos determinados por diferentes defectos enzimáticos, pero con características fenotípicas semejantes entre ellos ${ }^{3}$.

La mucopolisacaridosis tipo II (MPS II), o síndrome de Hunter, es una enfermedad de depósito lisosomal heredada siendo la única entre las MPS con un patrón recesivo ligado al cromosoma X. Afecta primariamente a los varones y su incidencia global ha sido estimada en 1 por cada 170000 varones nacidos vivos ${ }^{4,5}$; siendo la prevalencia en los judíos el doble de lo reportado en otras poblaciones ${ }^{6,7,8}$. En nuestro país no se conoce la incidencia de la MPS II.

El gen de la iduronato-2-sulfatasa (I2S) se localiza en Xq28 y se han descrito más de 300 mutaciones $^{2}$, esta enzima es requerida en el primer paso del metabolismo de GAG, heparan sulfato y dermatan sulfato y su deficiencia genera afectación multiorgánica y muerte temprana por acumulación tisular progresiva de los glicosaminoglicanos, contribuyendo a los signos y síntomas de la enfermedad ${ }^{9}$.

En general, las características clínicas no son evidentes al nacimiento, aunque tienden a ser pesados, algunos tienen hernias inguinales o umbilicales y hay una mayor incidencia de manchas mongólicas azules ${ }^{10}$. La edad al momento del diagnóstico suele ser de entre 18 y 36 meses $^{11}$, apareciendo características propias de la historia natural de la enfermedad como talla baja, displasia ósea, regresión psicomotriz, otitis, obstrucción nasal, hepatoesplenomegalia, macroglosia, rigidez en las articulaciones y contracturas, disfunción pulmonar, la ampliación de miocardio y disfunción valvular y compromiso neurológico. Además, presenta facies tosca, con labios gruesos, boca abierta y macrocefalia puente nasal aplanado ayudan a hacer el diagnóstico ${ }^{1,2,4,9,12}$.

El diagnóstico se basa en los antecedentes familiares, hallazgos clínicos y radiológicos, cuya confirmación se realiza mediante biopsia de piel y en el screening metabólico en orina con los resultados positivos a los test bromocetitrimeilamonio y azul de toluidina ${ }^{13}$, confirmándose con el dosaje de la enzima iduronatesulfatase, al estar esta disminuida ${ }^{1,9,14}$.
La enfermedad tiene curso progresivo pudiendo presentando discapacidad física, sensorial e intelectual afectando su calidad de vida ${ }^{14}$ por lo que estos niños necesitan un tratamiento integral con un equipo multidisciplinario debido a que su muerte ocurre generalmente hasta la primera o segunda década de la vida, por lo general a causa de obstrucción de vías respiratorias y/o insuficiencia cardiaca asociada con la pérdida de la función neurología².

La terapia de reemplazo enzimático (TRE) ha surgido como un nuevo tratamiento para los trastornos de las mucopolisacaridosis, incluyendo el síndrome de Hunter; demostrando que la terapia de reemplazo enzimático con idursulfasa es eficaz en relación con la capacidad funcional, los volúmenes de hígado y bazo y la orina excreción de glucosaminoglucanos en estos pacientes, mejorando su calidad de vida $9,15,16$.

El propósito de este reporte, es presentar una revisión y discutir una experiencia clínica de mucopolisacaridosis Il para los profesionales con la esperanza de que esa información ayudará a identificar niños afectados antes del curso de su enfermedad.

\section{CASO CLÍNICO}

Paciente varón de 7 años que es traído a emergencia del hospital por presentar marcada dificultad respiratoria 3 días previos caracterizados por disnea, sibilantes predominantemente nocturnos, tos con expectoración blanca intermitente sin olor, no fiebre, con agitación al movimiento; se trató con nebulizaciones con salbutamol de $2.5 \mathrm{mg}$ cada 4 horas presentando poca mejoría clínica. Un día antes del ingreso la disnea evoluciona provocándose a moderados esfuerzos, y se añade hipoactividad y somnolencia. El paciente tiene antecedente de mucopolisacaridosis tipo II, diagnosticado al año y 4 meses de vida. A los 9 meses de edad inicia la sospecha de una enfermedad congénita del metabolismo, tras detectar un perímetro cefálico mayor del p97, asociado a retraso de los hitos del desarrollo y una facies dismórfica hunteriana. Confirmándose con un screening metabólico en orina y un análisis de actividad enzimática.

Nació con 4 kg, el parto fue distócico por cesárea, por disminución de los movimientos fetales y su Ilanto no fue espontaneo. Tuvo retraso del desarrollo psicomotor con control cefálico a los 5 meses, control del tronco a los 8 meses, gateo a los 12 meses y marcha torpe con ayuda a los 2 años.

A los 2 años fue operado por hernia umbilical e inguinal izquierda. Presenta desde los 3 meses cuadros 
recurrentes de bronconeumonía. Inició su escolaridad a los 3 años en un colegio de educación especial. Tiene un primo de línea materna diagnosticado con mucopolisacaridosis tipo II, un hermano aparentemente sano de 3 años y madre de 38 años actualmente está embarazada.

Al examen físico se le encuentra agitado en aparente mal estado general. Presentaba taquicardia (100 lat/ min), taquipnea (50/min) ; estaba pálido, afebril $\left(37,5^{\circ} \mathrm{C}\right)$, con presión arterial de 90/60 mmHg. Su peso era 31 kg; su talla $126 \mathrm{~cm}$. Se observaron dismorfismo facial, piel gruesa, macrocefalia y macroglosia. En tórax se auscultaron sibilantes difusos inspiratorios y espiratorios, murmullo vesicular disminuido y crepitos bibasales. Paciente despierto, hipoactivo, se comunica con gestos y no emite palabra. Neurología lo evalúa encontrando una marcha con torpeza motora con ampliación de la base de sustentación, y un tono y trofismo ligeramente aumentados. Psiquiatría le diagnostica retraso mental severo y psicología trastorno del lenguaje y trastorno del desarrollo psicológico. Otorrinolaringología le da el diagnostico de hipertrofia adenoidea y oftalmología conjuntivitis atópica.

Las pruebas de laboratorio incluyeron el sreening metabólico en orina con resultados positivos a los test de bromocetirimeilmalonino y azul de toloudina. El examen de actividad enzimática reveló el I2S, marcador de MPS tipo II, disminuido: $1.5 \mathrm{nmol} / \mathrm{h} / \mathrm{ml}$ (VN: $12-23 \mathrm{nmol} / \mathrm{ml}$ ) siendo los valores enzimáticos para los otros tipos de MPS normales. Se halló en la radiografía de columna cervical lordosis y de columna lumbar, escoliosis derecha; en la ecografía abdominorenal, hepatoesplenomegalia y en la TAC se senos paranasales, hipertrofia adenoidea e hipertrofia de cornetes inferiores.

Se trata al paciente con bromuro de ipatropio, salbutamol, prednisona y gotas oftálmicas; y evoluciona favorablemente a la intercurrencia actual. Dado que su enfermedad es sistémica y progresiva, recibe manejo multidisciplinario. Actualmente se encuentra en estudios para inicio de tratamiento con reemplazo enzimático.

\section{DISCUSIÓN}

La Mucopolisacaridosis pertenece a un grupo de enfermedades hereditarias caracterizada por la actividad defectuosa de las enzimas lisosomales responsables de la degradación de mucopolisacáridos. La acumulación de glucosaminoglucanos en los lisosomas es responsable de las manifestaciones

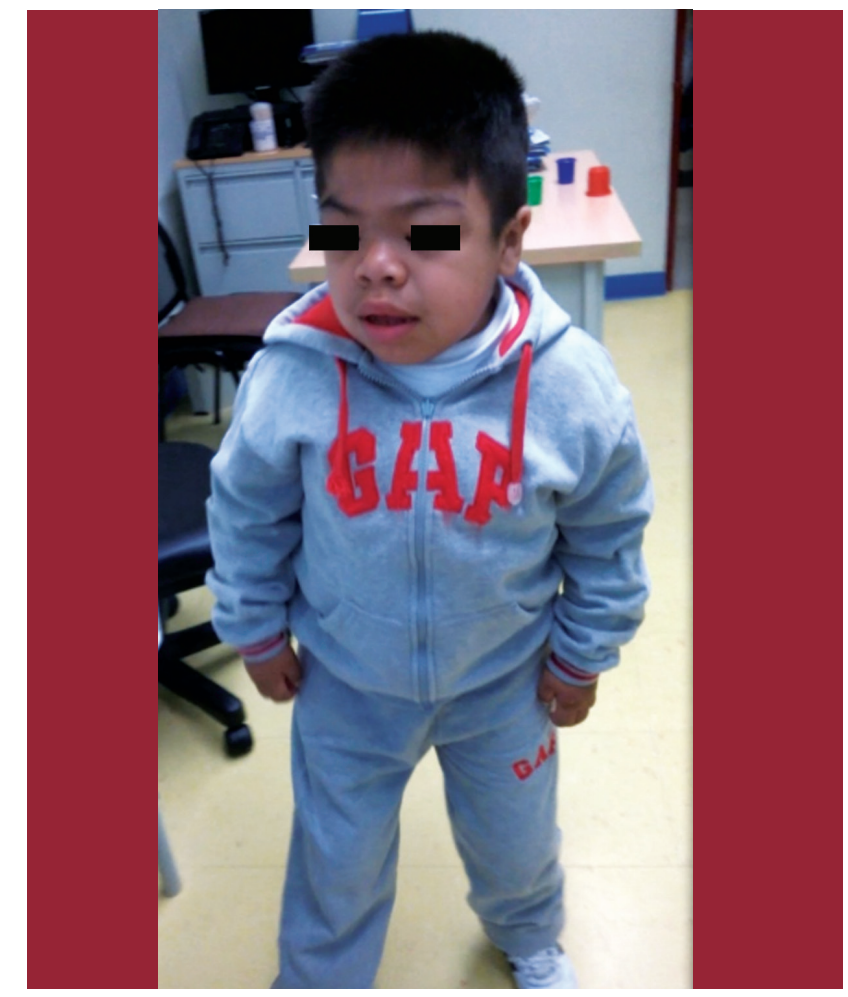

Figura 1. Paciente a los 8 años de edad, se aprecia macrocefalia, facie dismórfica hunteriana y mano en garra.

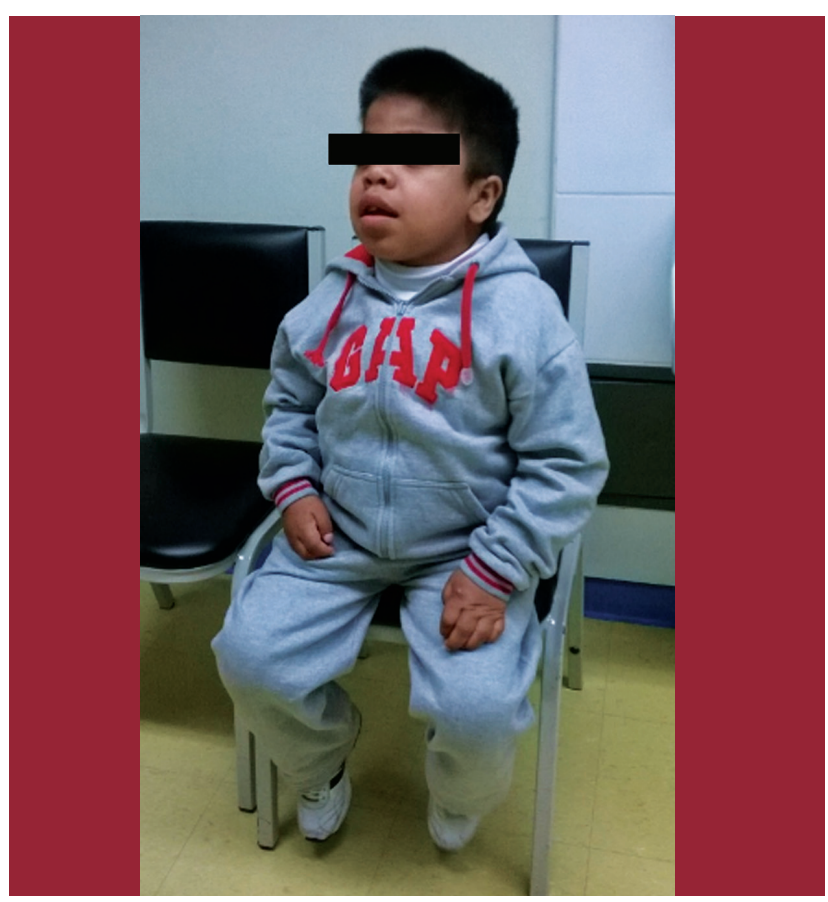

Figura 2. Paciente con mano en garra, talla corta y abdomen distendido debido a la hepatoesplenomegalia.

clínicas de este trastorno La Mucopolisacaridosis tipo II o también conocida como síndrome de Hunter es una entidad rara y es causada por una deficiencia de iduronato -2-sulfatasa. De todas las mucopolisacaridosis esta es la única con herencia ligada al cromosoma $\mathrm{X}^{22}$.

La MPS tipo II se clasifica en una forma atenuada 
y una forma severa. Esta clasificación se basa en la duración de la supervivencia, el pronóstico y la presencia o ausencia de compromiso en el sistema nervioso central (SNC). Los pacientes aparentan ser fenotípicamente normales al nacimiento. Sin embargo, en la forma grave las características clínicas aparecen alrededor de los dos a tres años de edad, mientras que en la forma leve las características clínicas aparecen más tardíamente ${ }^{23}$. En la forma severa existe grave retraso mental y retraso del desarrollo. La muerte generalmente ocurre en la primera o segunda década de la vida y la principal causa de muerte es causada por la enfermedad pulmonar obstructiva o la insuficiencia cardiaca, siendo las dos manifestaciones crónicas de la enfermedad de fondo 9 . Dentro de los hallazgos encontrados, nuestro paciente entraría dentro la forma severa de la enfermedad debido al tiempo de aparición de los síntomas, el retraso mental y psicomotor presente, además de la enfermedad obstructiva crónica como dato de reingreso frecuente, lo cual podría influir en su pronóstico a largo plazo de manera negativa.

El diagnóstico de esta patología se hace generalmente por la presentación clínica y la evaluación de las deformidades óseas. Sin embargo, ya desde el nacimiento hay características iniciales que al concordar con los hallazgos posteriormente realizados hacen concordar con el diagnostico final. Se menciona que en niños afectados por la forma severa de esta enfermedad, pueden presentar un peso elevado al nacimiento como primera manifestación de la enfermedad ${ }^{18}$, lo cual concuerda con los 4 kilos encontrados en el paciente en su primera evaluación post natal.

Posteriormente con el transcurso de la enfermedad, las presentaciones clínicas más comunes son la macrocefalia (mejor definida como una dolicocefalia debida a un engrosamiento de la bóveda craneana), procesos del tracto respiratorio superior a repetición, obstrucción nasal, retraso mental de intensidad variada, rasgos faciales toscos, hipertrofia adenoidea, visceromegalia e hipotonía²; todas las cuales estaban presentes durante toda la historia de la enfermedad del caso presentado.

Las manifestaciones radiológicas son bastante compatibles compatibles con el desarrollo de la enferemedad,endondela revisión bibliográficamenciona hallazgos sugestivos como la presencia de dolicocefalia, alteraciones en la morfología de la silla turca, alteraciones de los cuerpos vertebrales, cifoescoliosis y presencia de huesos cárpales de características anómalas ${ }^{20,21}$. Muchos de los cuales son también de importancia clínica, pues en muchos casos estas alteraciones morfológicas son de carácter incapacitante e influyen en la la posterior evolución del paciente.

Por otro lado, la aparición progresiva del retraso e involución del desarrollo, no es de menos importancia, ya que en muchos casos pueden ser las primeras manifestaciones enfermedades de este tipo, y los cuales fueron parte de este caso al evidenciarse un retraso del desarrollo psicomotor, muy probablemente debido a la afectación tanto neurológica como a las deformidades óseas derivadas de su enfermedad de base ${ }^{18}$.

Adicionalmente, la literatura menciona que las complicaciones cardiovasculares son bastante frecuentes, siendo la manifestación mas frecuente el compromiso valvular. Sin embargo, nuestro paciente no ha presentado síntoma cardiovascular alguno en el transcurso de la enfermedad. No obstante, sí presenta un seguimiento estricto por cardiología para la detección precoz de eventualidades ${ }^{12}$.

Unavezdeterminadalasospechaclínica,esconveniente la realización de exámenes complementarios cuyo resultado apoye fuertemente el diagnóstico. El examen complementario más sencillo de realizar es la investigación de la eliminación aumentada de GAG por la orina ${ }^{17}$. Es preciso recordar que existe una relación inversa entre la edad del paciente y la cuantía de la eliminación, de tal modo que en los primeros meses de vida es siempre superior que en edades posteriores, y es necesario conocer los valores normales para cada edad. El método más sencillo, el test de Berry o de azul de toluidina, es de carácter "cualitativo» e identifica el cambio de coloración que se produce al poner en contacto una gota de orina fresca en papel de filtro, que se tiñe con azul de toluidina en medio ácido. En nuestro caso, la muestra fue tomada de manera precoz e inmediatamente luego de la primera sospecha, por lo que el resultado positivo apoyo el diagnostico. Posteriormente, se realizo de un estudio enzimático del I2S y estudios genéticos que corroboraron la sospecha diagnostica, para de esta manera seguir los algoritmos diagnostico de esta enfermedad y asi descartar los otros tipos de mucopolisacaridosis ${ }^{22}$.

Hasta hace poco tiempo, el tratamiento del síndrome de Hunter era paliativo, centrándose solo en signos y síntomas. De los muchos métodos probados para tratar la enfermedad, como el trasplante de médula ósea, terapia genética, trasplante de fibroblastos solo la terapia de reemplazo enzimático intravenoso con enzima humana recombinante $12 \mathrm{~S}$ ha sido probada en ensayos clínicos. La enzima idursulfasa (Elaprase, Shire 
Human Genetic Therapies, Inc, Cambridge, MA) fue recientemente aprobada para su uso en Norteamérica y la Unión Europea, basado en un ensayo clínico que demostró que pacientes tratados por un año con $0.5 \mathrm{mg} / \mathrm{kg}$ de idursulfasa semanal mejoraron notablemente en relación a los que recibieron placebo. La dosis de $0.5 \mathrm{mg} / \mathrm{kg}$ fue aprobada para el tratamiento ${ }^{9-15-19}$. En el presente caso, el paciente se encuentra en evaluación para la posibilidad de recibir tratamiento enzimático en el futuro.

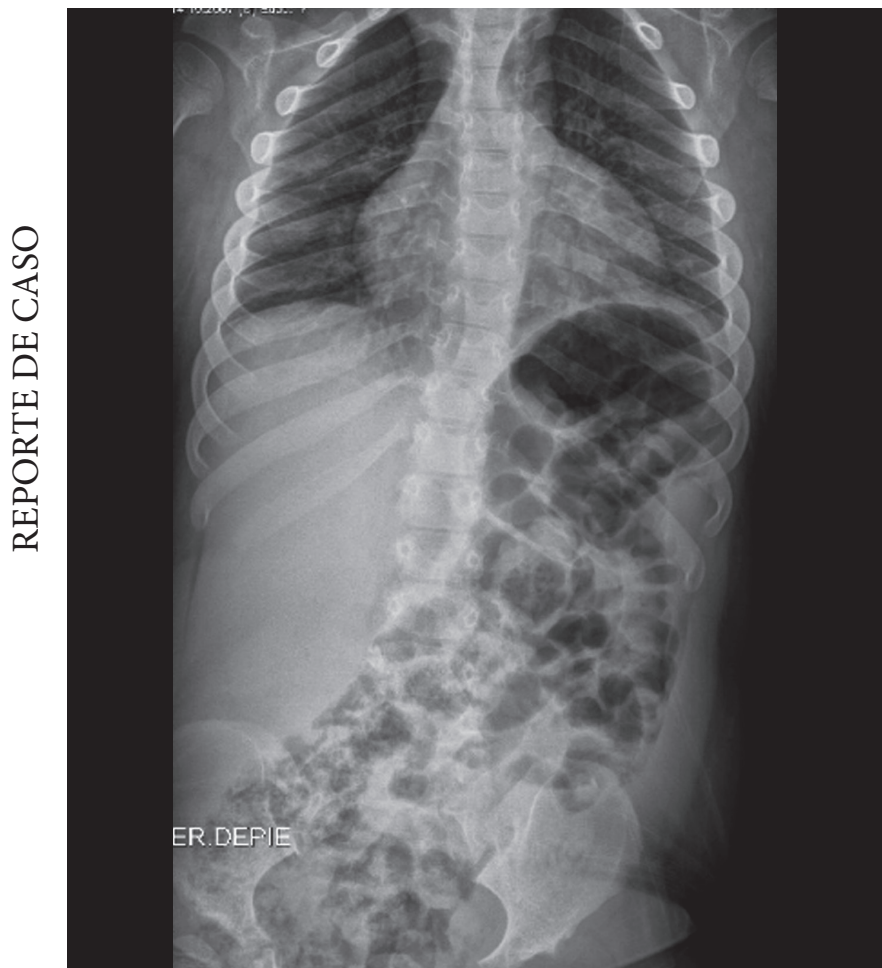

Figura 3. Radiografía de tórax y abdomen donde se observa escoliosis.

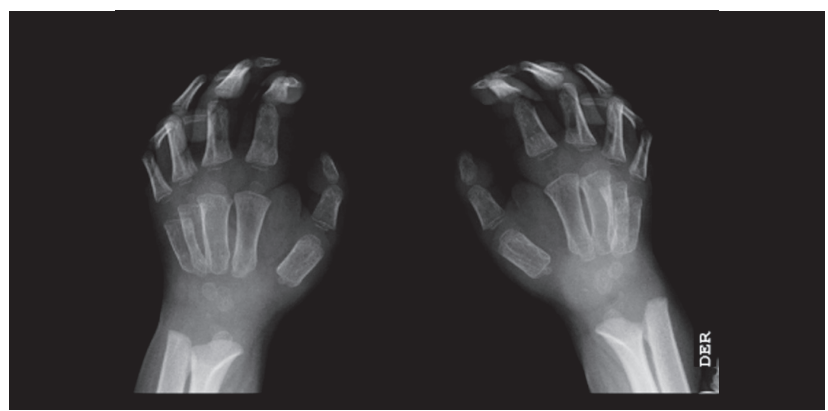

Figura 4. Radiografía de mano, se observa mano en garra.

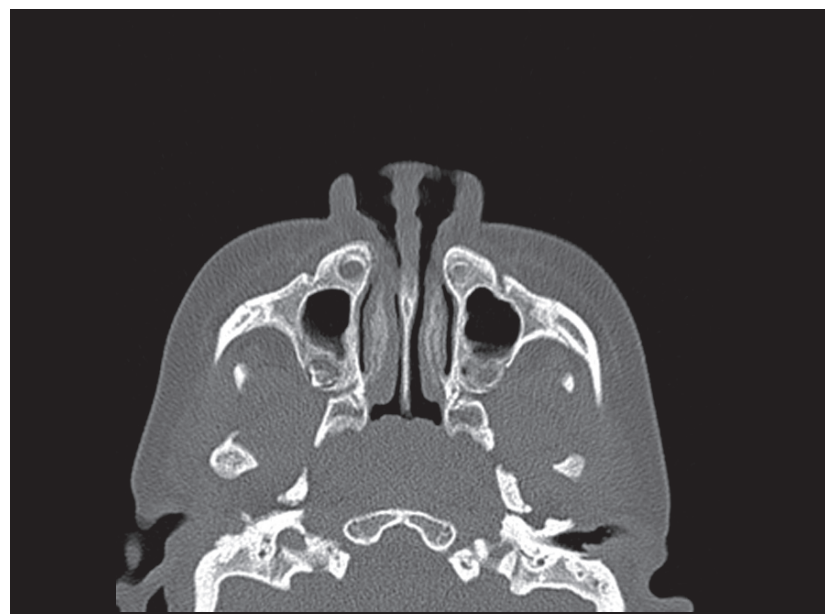

Figura 5. Tomografía de cavum, se observa hipertrofia adenoidea.

\section{Financiamiento: Autofinanciado.}

Conflicto de interés: Los autores declaran no tener conflictos de interés en la publicación de este artículo.

Recibido: 17 de marzo del 2017

Aprobado: 30 de marzo del 2017

\section{REFERENCIAS BIBLIOGRÁFICAS}

1. Nelson Textbook of Pediatrics. 18th ed. Philadelphia: Saunders; 2007. 620-6 p.

2. Martin R, Beck M, Eng C, Giugliani R, Harmatz P, Muñoz V, et al. Recognition and diagnosis of mucopolysaccharidosis II (Hunter syndrome). Pediatrics. febrero de 2008;121(2):e377-86.

3. Maquetación 1-protocolo_de_actuacion_en_las_mucopolisacaridosis. pdf [Internet]. [citado 25 de septiembre de 2015]. Recuperado a partir de: http://www.aeped.es/sites/default/files/documentos/protocolo_de_ actuacion_en_las_mucopolisacaridosis.pdf

4. Baehner F, Schmiedeskamp C, Krummenauer F, Miebach E, Bajbouj M, Whybra $C$, et al. Cumulative incidence rates of the mucopolysaccharidoses in Germany. J Inherit Metab Dis. 2005;28(6):1011-7.

5. Poorthuis BJ, Wevers RA, Kleijer WJ, Groener JE, de Jong JG, van Weely $\mathrm{S}$, et al. The frequency of lysosomal storage diseases in The Netherlands. Hum Genet. agosto de 1999;105(1-2):151-6.

6. Zlotogora J, Schaap T, Zeigler M, Bach G. Hunter syndrome in Jews in Israel: further evidence for prenatal selection favoring the Hunter allele. Hum Genet. marzo de 1991;86(5):531-3.
7. Schachern PA, Shea DA, Paparella MM. Mucopolysaccharidosis I-H (Hurler's syndrome) and human temporal bone histopathology. Ann Otol Rhinol Laryngol. febrero de 1984;93(1 Pt 1):65-9.

8. Ben Simon-Schiff E, Bach G, Zlotogora J, Abeliovich D. Combined enzymatic and linkage analysis for heterozygote detection in Hunter syndrome: identification of an apparent case of germinal mosaicism. Am J Med Genet. 1 de noviembre de 1993;47(6):837-42.

9. Wraith JE, Scarpa M, Beck M, Bodamer OA, De Meirleir L, Guffon N, et al. Mucopolysaccharidosis type II (Hunter syndrome): a clinical review and recommendations for treatment in the era of enzyme replacement therapy. Eur J Pediatr. marzo de 2008;167(3):267-77.

10. Su F., Li F., Jin H.Z. Extensive Mongolian spots in a child with mucolipidosis II. 2010. 49:438-40.

11. Cleary MA WJ. The presenting features of mucopolysaccharidosis type IH (Hurler syndrome). 84. 1995;337-9.

12.Gajula P, Ramalingam K, Bhadrashetty D. A rare case of mucopolysaccharidosis: Hunter syndrome. J Nat Sci Biol Med. 2012;3(1):97-100. 
13. RM031-2015-MINSA.pdf [Internet]. [citado 26 de septiembre de 2015]. Recuperado a partir de: http://www.minsa.gob.pe/dgsp/documentos/ Guias/RM031-2015-MINSA.pdf

14. Guia Hunter.mail.pdf [Internet]. [citado 26 de septiembre de 2015]. Recuperado a partir de: http://www.mpsesp.org/portal1/images/ content/Guia\%20Hunter.mail.pdf

15. da Silva EMK, Strufaldi MWL, Andriolo RB, Silva LA. Enzyme replacement therapy with idursulfase for mucopolysaccharidosis type II (Hunter syndrome). Cochrane Database Syst Rev. 2014;1:CD008185.

16. Alcalde-Martín C, Muro-Tudelilla JM, Cancho-Candela R, GutiérrezSolana LG, Pintos-Morell G, Martí-Herrero M, et al. First experience of enzyme replacement therapy with idursulfase in Spanish patients with Hunter syndrome under 5 years of age: case observations from the Hunter Outcome Survey (HOS). Eur J Med Genet. diciembre de 2010;53(6):371-7.

17.Baldellou Vasquez A., Garcia Jimenez M.J. Diagnóstico de la mucopolisacaridosis II (síndrome de Hunter) en atención primaria. Acta
18. Gauri Shankar Shah, Tania Mahal, Subodh Sharma. Atypical clinical presentation of mucopolysaccharidosis type II (Hunter syndrome): a case report. Journal of Medical Case Reports 2010, 4:154

19. Muenzer J, Wraith JE, Beck M, et al. A phase II/III clinical study of enzyme replacement therapy with idursulfase in mucopolysaccharidosis II (Hunter syndrome) [published correction appears in Genet Med. 2006;8(9):599]. Genet Med. 2006; 8(8):465-473

20. Palmucci Stefano et al. Imaging findings of mucopolysaccharidoses: a pictorial review Insights Imaging (2013) 4:443-459.

21. RASALKAR, DD et al. Pictorial review of mucopolysaccharidosis with emphasis on MRI features of brain and spine. The British Journal of Radiology, 84 (2011), 469-477

22. E. Guillén-Navarro et al. Guía de práctica clínica para el tratamiento del síndrome de Hunter. Med Clin (Barc). 2013;141(10):453.e1-453.e13

23. Guelbert, Norberto y col. Guía para el diagnóstico, seguimiento y tratamiento de la mucopolisacaridosis de tipo II (MPS-II) o Enfermedad de Hunter. Arch Argent Pediatr 2011;109(2):175-181

Consulte la Versión Electrónica de la Revista:

Facultad de Medicina Humana Universidad Ricardo Palma

http://revistas.urp.edu.pe/index.php/RFMH<smiles>C1=CC[In]=C1</smiles>

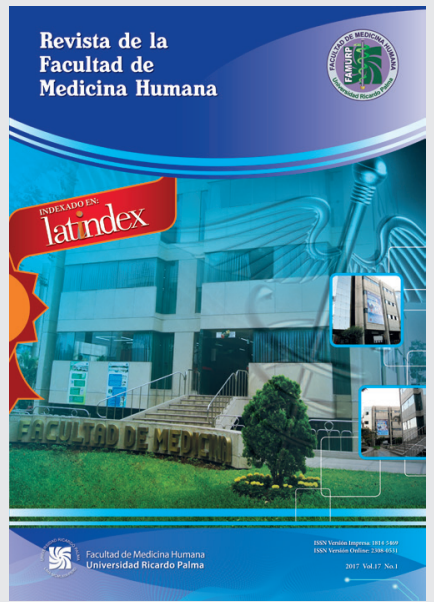

